\title{
Involvement of EZH2 in aerobic glycolysis of prostate cancer through miR-181b/HK2 axis
}

\author{
TAO TAO $^{1^{*}}$, MING CHEN $^{2 *}$, RANRAN JIANG ${ }^{3 *}$, HAN GUAN $^{2}$, \\ YEQING HUANG ${ }^{2}$, HUAN SU ${ }^{2}$, QIANG $\mathrm{HU}^{2}, \mathrm{XU}^{\mathrm{HAN}}{ }^{2}$ and JUN XIAO ${ }^{1}$ \\ ${ }^{1}$ Department of Urology, Anhui Provincial Hospital, Anhui Medical University, Hefei, Anhui 230001; \\ ${ }^{2}$ Department of Urology, Zhongda Hospital, Medical School, Southeast University, Nanjing, Jiangsu 210009; \\ ${ }^{3}$ Jiangsu Key Laboratory of Biological Cancer Therapy, Xuzhou Medical University, Xuzhou, Jiangsu 221002, P.R. China
}

Received September 24, 2016; Accepted February 3, 2017

DOI: $10.3892 /$ or.2017.5430

\begin{abstract}
Recent studies suggest that several types of tumors preferentially metabolize glucose through aerobic glycolysis, a phenomenon known as the Warburg effect. However, it remains largely unexplored whether metabolic reprogramming is involved in prostate cancer ( $\mathrm{PCa}$ ) progression. In this study, we found that histone methyltransferase enhancer of zeste homolog 2 (EZH2) dysregulated in PCa development regulated cellular growth and aerobic glycolysis through miR-181b/hexokinase 2 (HK2) axis. Aberrant expression profiles of coding RNA and microRNA were examined by two large, independent clinical prostate cancer data sets. The results indicated that $\mathrm{EZH} 2$ expression was elevated followed by PCa development. A set of glycometabolism-related genes were positively correlated with EZH2 expression such as HK2. The depletion of EZH2 in cell experiments inhibited PCa cell growth and aerobic glycolysis accompanying the upregulation of miR-181b. Western blot and luciferase reporter assays showed that miR-181b inversely modulated HK2 by directly targeting the binding site within 3'-untranslated regions. Moreover, decreased miR-181b expression largely abrogated the effect of sh-EZH2 on HK2 expression and HK2-induced glucose metabolism process. Immunohistochemistry (IHC) and in situ hybridisation (ISH) analysis further revealed a significant correlation in EZH2, miR-181b and HK2 expression in nude mouse tumor xenograft. Taken together, these findings provide the first evidence that EZH2/miR-181b/HK2 pathway plays a positive role in PCa development. Targeting
\end{abstract}

Correspondence to: Professor Jun Xiao, Department of Urology, Anhui Provincial Hospital, Anhui Medical University, Hefei, Anhui 230001, P.R. China

E-mail: ahurology@126.com

"Contributed equally

Key words: enhancer of zeste homolog 2, miR-181b, hexokinase 2, aerobic glycolysis, prostate cancer this aberrantly activated pathway may provide a new therapeutic strategy against $\mathrm{PCa}$.

\section{Introduction}

One of the most distinguishing characteristics between normal and tumorigenic cells is altered glucose metabolism, which is listed as one of the ten hallmarks of cancer (1). Under aerobic conditions, normal differentiated cells extract energy from glucose chiefly through oxidative phosphorylation, but tumor cells metabolize more glucose to lactate. This phenomenon is termed the Warburg effect (aerobic glycolysis) $(2,3)$. The preference to utilize glycolysis offers several advantages to cancer cells including adaptation in hypoxic and acidic environments (lactate production), which help cancer cells in invasion and apoptosis resistance (4-7). Additionally, increased glycolysis in cancer tissues allows the diversion of glycolytic intermediates into various biosynthetic pathways that can synthesize macromolecules and organelles required for the assembly of new cancer cells, which is important for tumor cell proliferation $(8,9)$. Until now, few studies on prostate cancer energy metabolism have been reported.

The high rate of glycolysis commonly observed in cancers is presumably ascribed to upregulation of key enzymes in glycolysis including hexokinase 2 (HK2), 5 glyceraldehyde-3-phosphate dehydrogenase (GAPDH), 6-phosphofructo-1-kinase (PFK1) $(10,11)$. HK2, coding for the first rate-limiting enzyme of glycolysis, is found to be overexpressed in some malignant tumors. As reported, HK2 could be bound to the outer mitochondrial membrane $(12,13)$. At this location, it helps couple ATP formation in mitochondria to the phosphorylation of glucose, thus conferring cancer cells with a highly glycolytic phenotype and ample biosynthetic precursor (14). In this study, we firstly reported that HK2 was elevated in prostate cancer tissues compared to prostatic hyperplasia tissues. Moreover, its expression significantly increased in castration-resistant prostate cancer (CRPC) compared to androgen-dependent prostate cancer (ADPC). Further study revealed that HK2 regulated genes primarily associated with glycometabolism and cell cycle progression in prostate cancer. In addition, the results showed that HK2 was directly regulated by $\mathrm{EZH} 2 / \mathrm{miR}-181 \mathrm{~b}$ axis at post-transcriptional 
level. EZH2/miR-181b axis promoted aerobic glycolysis by upregulating $\mathrm{HK} 2$ in prostate cancer.

\section{Materials and methods}

Cell culture and reagents. The human PCa cell line PC-3 was purchased from KeyGene Biotech (Nanjing, China). Cells were cultured in RPMI-1640 supplemented with $10 \%$ fetal bovine serum, $100 \mathrm{U} / \mathrm{ml}$ penicillin, and $100 \mu \mathrm{g} / \mathrm{ml}$ streptomycin at $37^{\circ} \mathrm{C}$ in a humidified atmosphere with $5 \% \mathrm{CO}_{2}$. Transfections of sh-EZH2, miR-181 mimics, As-miR-181b, and negative control (NC) (GenePharma; Shanghai, China) were performed with Lipofectamine 2000 (Invitrogen, Carlsbad, CA, USA).

Data mining and bioinformation analysis. The GSE35988 and GSE21032 datasets were downloaded from GEO database (http://www.ncbi.nlm.nih.gov/geo) and re-analyzed with $\mathrm{R}(15,16)$. Pearson's correlation was used to analyze the relationship between EZH2 and all identified genes with Matlab software $(\mathrm{P}<0.05)$. GSEA (http://www.broadinstitute.org/ gsea/) analysis was used to identify pathway gene sets that are correlated with the HK2 expression profile. The gene sets were derived from the Molecular Signatures Database (http://www. broadinstitute.org/gsea/msigdb/index.jsp).

Oligonucleotides and cell transfection. The EZH2-shRNA and HK2-shRNA oligonucleotide were cloned into vectors U6/GPF/Neo by GenePharma. A control shRNA unrelated to any human sequence was used as the negative control (NC). Oligonucleotides were chemically synthesized by GenePharma according to the sequences of hsa-miR-181b mimic (miR181b), 5'-AACAUUCAUUGCUGUCGGUGGGU-3'; hsa-miR-181b antisense oligonucleotide (As-miR-181b), 5'-ACCCACCGACAGCAATGAATGTT-3'; scrambled miRNA mimic, 5'-UUCUCCGAACGUGUCACGUTT-3'; scrambled microRNA inhibitor, 5'-CAGUACUUUUGUGUA GUACAA-3'. Cells at $60-70 \%$ confluence were transfected using Lipofectamine 2000 reagent (Invitrogen). The oligonucleotides and plasmids formed transfection complexes with Lipofectamine 2000 reagent. The transfection complexes were added to $\mathrm{PCa}$ cells at different concentrations and then incubated for $6-8 \mathrm{~h}$ before changing the medium. The lentiviral vector containing EZH2-siRNA and NC sequence was generated by GeneChem Inc.

Colony formation assay. Cells were plated onto dishes in RPMI-1640 culture. After 48 h of EZH2-shRNA processing, the cells were washed thrice with phosphate-buffered saline (PBS), and fresh broth was supplied. After two weeks, the cells were fixed in $5 \mathrm{ml}$ of $4 \%$ paraformaldehyde for $15 \mathrm{~min}$. Giemsa staining was performed for $20 \mathrm{~min}$, and the cells were washed thrice with PBS. The clone number was counted under a microscope.

Glycolysis stress test. The extracellular acidification rate (ECAR) was measured using the Seahorse XF96 Analyzer Glycolysis which calculates the net production and extrusion of protons into the extracellular medium. As glycolysis occurs, the resulting acidification of the medium surrounding the cells is measured directly by the XF analyzer and reported as the
ECAR. Initially, cells are incubated in glycolysis stress test medium without glucose. The ECAR refers to non-glycolytic acidification, which includes $\mathrm{CO}_{2}$ evolution followed by its hydration to carbonic acid and bicarbonate, as well as proton extrusion. The first injection is a saturating concentration of glucose. Glucose is taken up by the cells and catabolized to lactate, producing ATP and protons, with a corresponding rapid increase in ECAR. This glucose-induced response is reported as the rate of glycolysis under basal conditions. The second injection is oligomycin. It inhibits mitochondrial ATP production and thus shifts the energy production to glycolysis, with the increase in ECAR revealing the maximum glycolytic capacity. The final injection is 2-DG, a glucose analog, which inhibits glycolysis through competitively binding to glucose hexokinase. The resulting decrease in ECAR further confirms that the ECAR produced in the experiment is due to glycolysis.

Quantitative RT-PCR. RNA was extracted from cells or tissues using TRIzol (Invitrogen). miR-181b (qRT-PCR) reactions were performed using Fermentas reverse transcription reagents and SYBR Green PCR Master Mix (GenePharma) according to manufacturer's protocols. U6 was used for normalisation. Relative gene expression was calculated by the $2^{-\Delta \Delta C t}$ method.

Western blot analysis. Western blot analysis was performed as previously described (17). Immunoblot was performed using appropriate primary antibodies: EZH2 (1:1000; Cell Signaling Technology, Inc., Danvers, MA, USA), HK2 (1:1000; Abcam, Cambridge, UK), and $\beta$-actin (1:1000; Santa Cruz Biotechnology; Santa Cruz, CA, USA).

Luciferase reporter assay. An HK2 3'UTR-Luc reporter was created by ligating the HK2 3'UTR PCR product into the XbaI site of the pGL3 control vector (Invitrogen). A mutant reporter was generated from pGL3-WT-HK2 3'UTR-Luc by deleting the binding site for miR-181b 'UGAAUGU'. A $1.9 \mathrm{~kb}$ fragment upstream of human pri-miR-181b stem-loop was constructed into pGL3-basic plasmids (Invitrogen). Luciferase activity was measured using the Dual-Luciferase Reporter Assay System (Promega, Madison, WI, USA).

Xenograft tumor assay. BALB/c nude mice at 4 weeks of age were purchased from the Animal Center of the Cancer Institute at the Chinese Academy of Medical Science. All mice were divided randomly into negative control group and EZH2 inhibition group. The guidelines for animal welfare were approved by the Ethics Committee on Animal Research of Southeast University. To test the effect of EZH2 on prostate cancer growth and downstream pathway in vivo, 4x10 6 PC-3 cells infected with EZH2-siRNA lentivirus were implanted into subcutaneous tissue of each nude mouse in EZH2 inhibition group. The tumor volumes were measured every 7 days by vernier calipers.

Immunohistochemical staining (IHC) and in situ hybridization (ISH). IHC and ISH were performed as previously described (18). IHC was performed using appropriate primary antibodies according to the manufacturer's instructions. Tissue sections were stained with 3'3-diaminobenzidine solution 

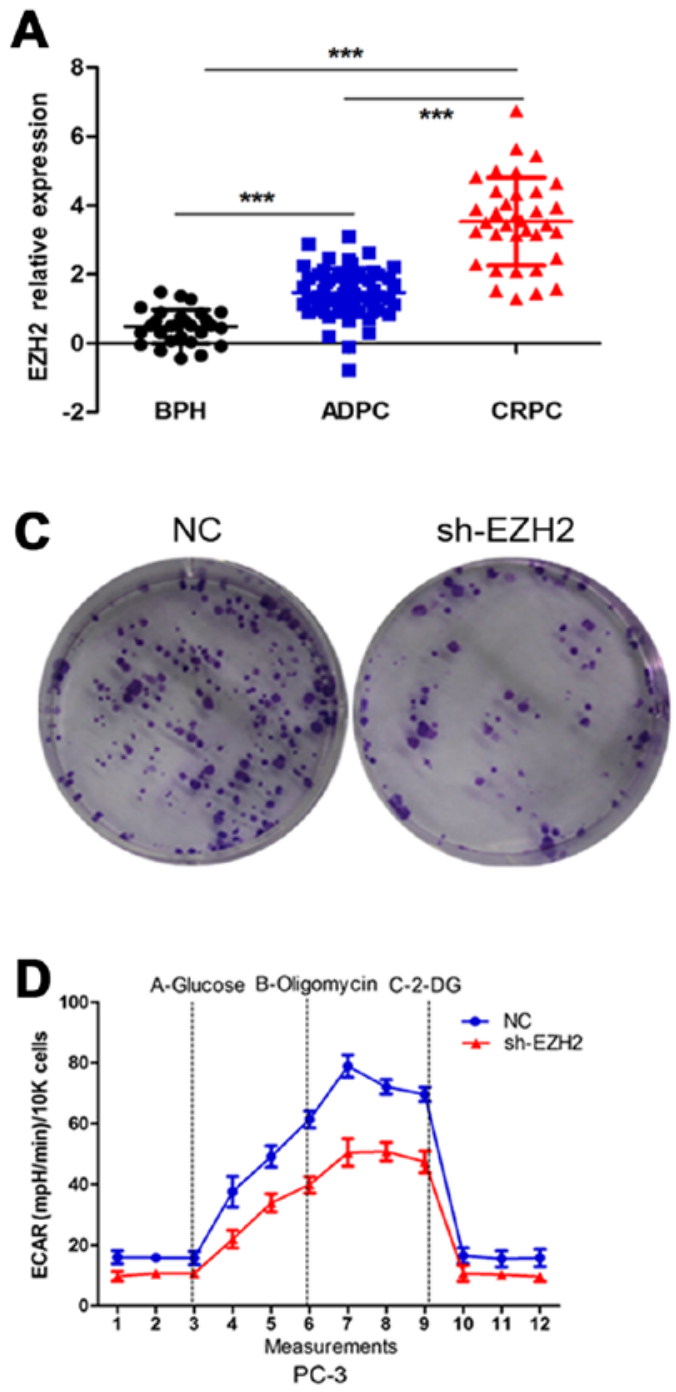
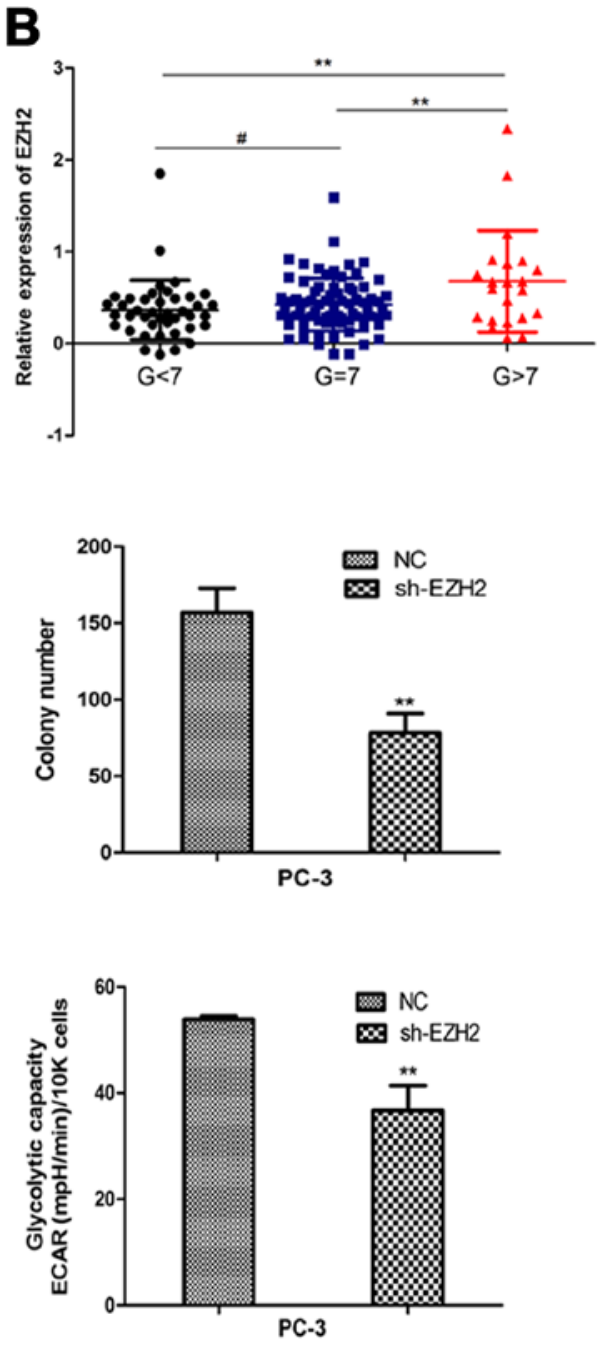

Figure 1. The expression and function of EZH2 in PCa tissues. (A) EZH2 expression was elevated in PCa tissues compared to BPH. Further, EZH2 expression significantly increased in CRPC compared to ADPC. (B) EZH2 expression was higher in Gleason $>7$ prostate cancer tissues compared to Gleason $\leq 7$ ones. (C) Colony formation assay revealed that EZH2 inhibition can significantly inhibit the number of cell colonies. (D) The glycolytic capacity was determined using the seahorse extracellular flux analyzer. ECAR was continuously calculated. ECAR following the addition of glucose defines glycolysis and ECAR following oligomycin represents maximum glycolytic capacity. The data showed that inhibition of EZH2 weakened the glycolytic capacities of PCa cells compared with negative control. ${ }^{\#} \mathrm{P}>0.05,{ }^{* *} \mathrm{P}<0.01,{ }^{* * * *} \mathrm{P}<0.001$.

(DAB). ISH was performed using antisense locked nucleic acid (LNA)-modified probes based on the sequence of hsamiR-181b (Boster, Wuhan, China). The hybridized probes were also detected by incubating with DAB.

Statistical analysis. Data were obtained from at least three independent experiments and presented as mean $\pm \mathrm{SD}$. The significance of differences was calculated using one-way ANOVA for three-group comparisons and t-tests for twogroup comparisons with SPSS 13.0 software package. Pearson's correlation analysis was performed using Matlab software. $\mathrm{P}<0.05$ was considered to indicate a statistically significant difference.

\section{Results}

EZH2 expression and function in PCa. Firstly, we employed the GEO database to analyze the expression of EZH2 in benign prostatic hyperplasia tissues and prostate cancer tissues in different stages and different pathological levels. The results indicated that EZH2 expression was elevated in prostate cancer tissues compared to prostatic hyperplasia tissues. Further, EZH2 expression significantly increased in CRPC compared to ADPC (Fig. 1A). Besides, EZH2 expression was higher in Gleason $>7$ prostate cancer tissues compared to Gleason $\leq 7$ ones (Fig. 1B). To further explore the effect of EZH2 on prostate cancer cells, EZH2 was downregulated by sh-EZH2. Cell tablet assays revealed that EZH2 inhibition can significantly inhibit the number of cell colonies (Fig. 1C). To assess the function of EZH2 in cellular energy metabolism, we performed a glycolysis stress test. Inhibition of EZH2 weakened the glycolytic abilities of prostate cancer cells compared with negative control (Fig. 1D).

EZH2 correlates with glycometabolism-related genes in PCa. To evaluate the potential mechanism in aberrant expression of EZH2 and cellular metabolism and growth, Pearson's correlations were used to analysis the relationship between EZH2 

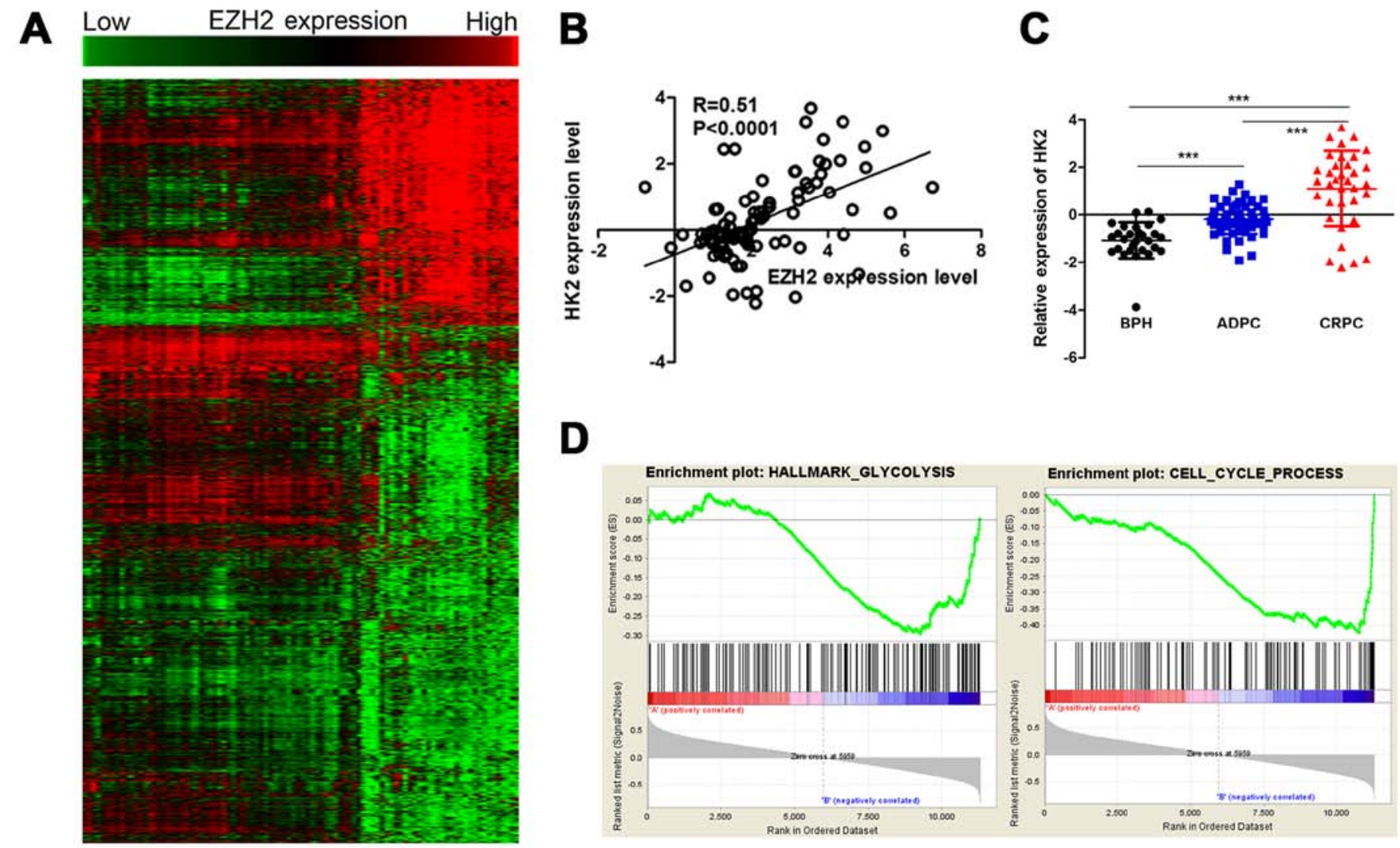

D

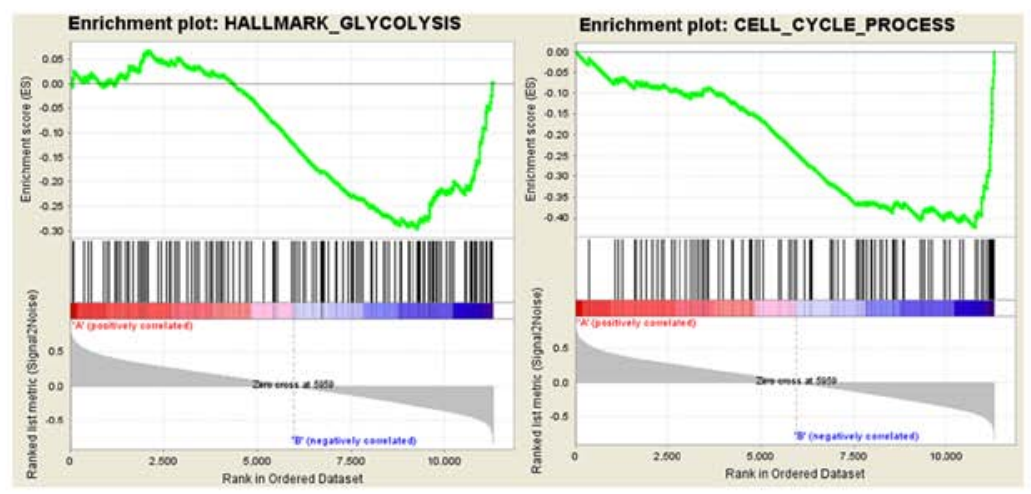

Figure 2. EZH2 correlates with glycometabolism-related genes in prostate cancer. (A) The heat map shows EZH2 specific gene signature included 925 genes positively correlated with EZH2 expression, and 1,952 genes negatively correlated with EZH2 expression. (B) Pearson's correlations analysis showed HK2 was positively correlated with EZH2 expression. (C) HK2 expression was elevated in PCa tissues compared to BPH. Moreover, HK2 expression significantly increased in CRPC compared to ADPC. (D) GSEA analysis revealed that genes correlated with HK2 were primarily associated with glycometabolism and cell cycle progression. ${ }^{* * *} \mathrm{P}<0.001$.

A

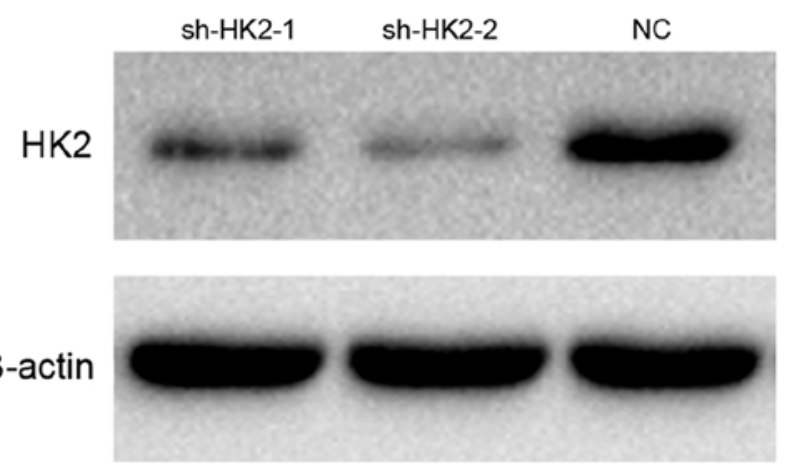

B
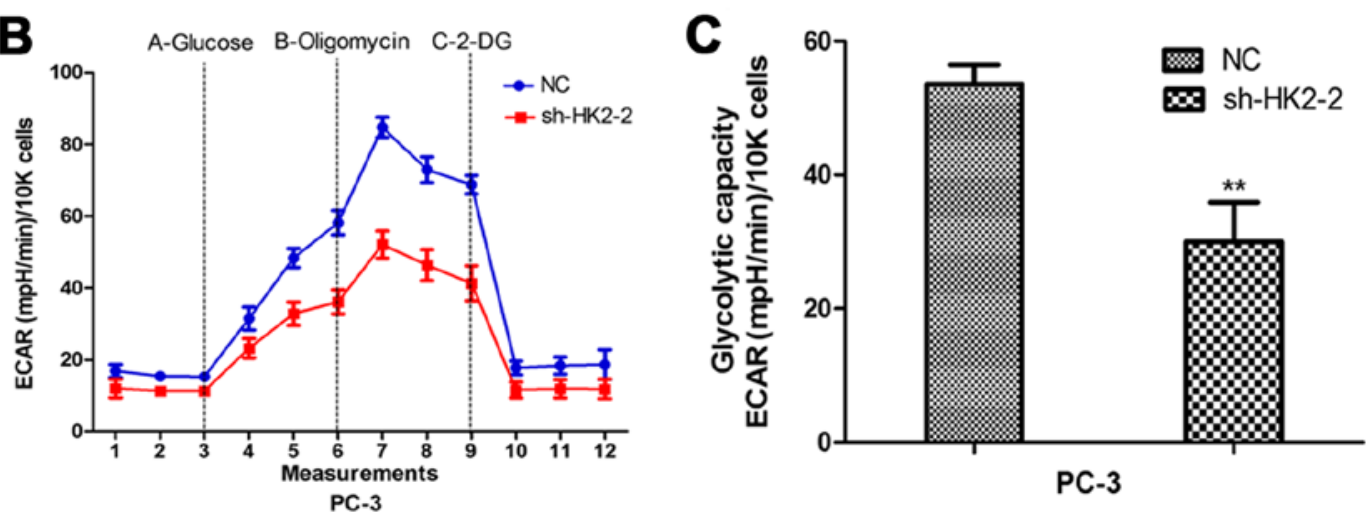

Figure 3. The biological function of HK2 in PCa cells. (A) Cells were treated with sh-HK2. The protein expression level of HK2 was detected by western blot assay. (B and C) Inhibition of HK2 significantly weakened the glycolytic capacities of PCa cells compared with negative control. ${ }^{* *} \mathrm{P}<0.01$. 
A

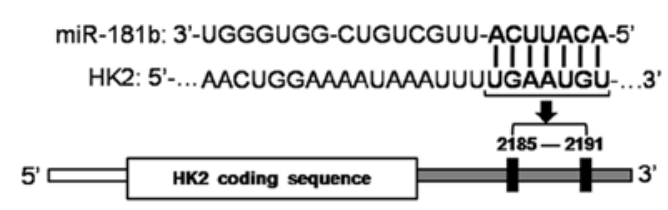

D
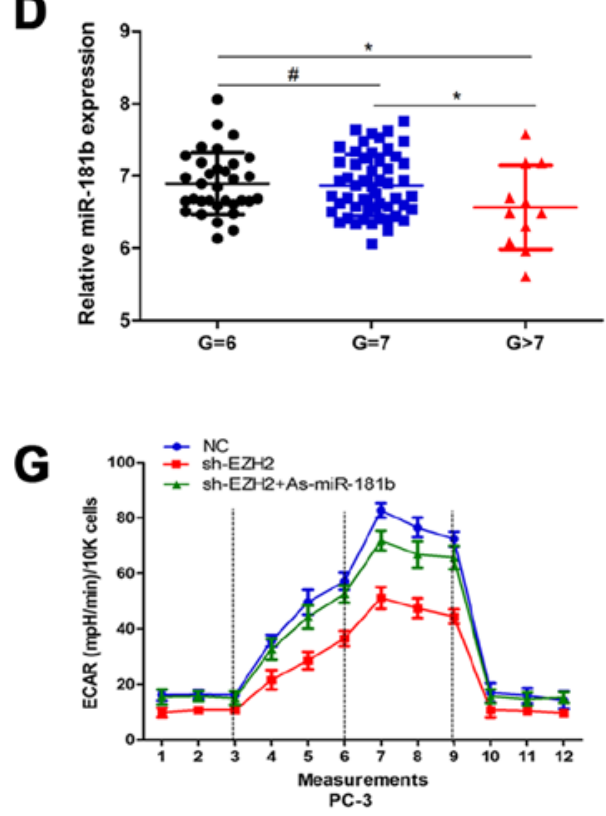

B

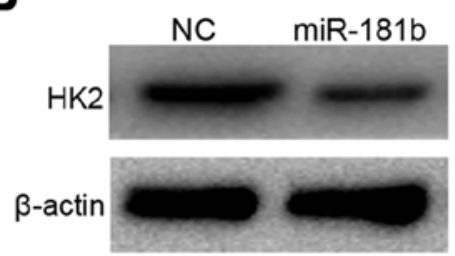

E
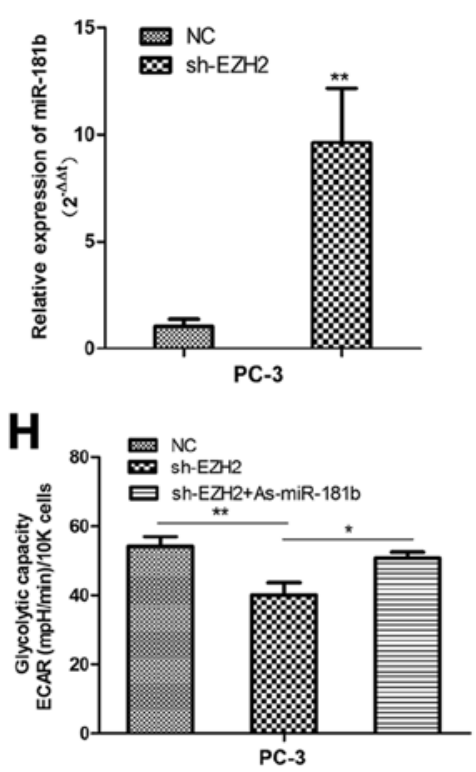

C

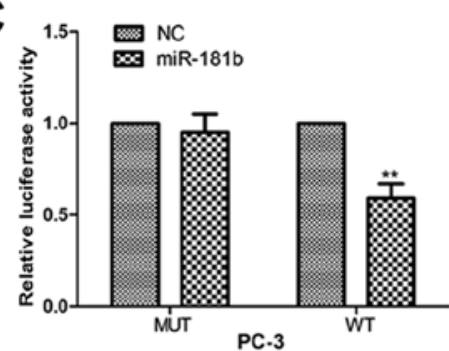

$\mathbf{F}$

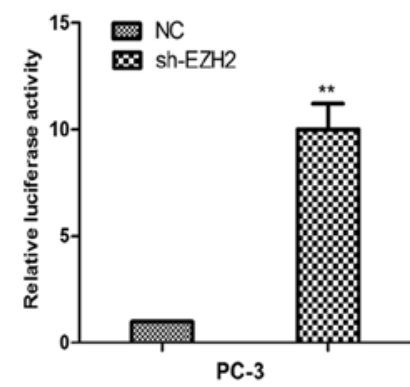

I

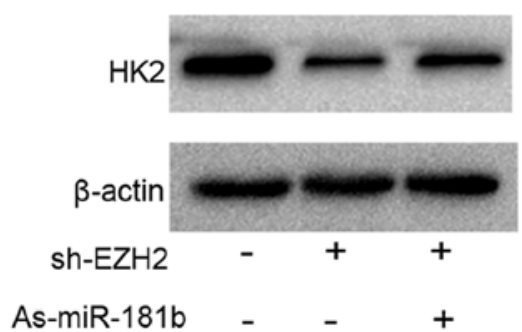

Figure 4. EZH2 promotes cellular glucose metabolism through miR-181b/HK2 axis. (A) Putative binding sites of miR-181b within the HK2 3'UTR, as predicted by the microRNA.org algorithms. (B) Overexpression of miR-181b inhibited HK2 expression at the protein level. (C) miR-181b downregulated the luciferase activity controlled by the wild-type HK2 3'UTR but did not affect the luciferase activity controlled by the mutant HK2 3'UTR. (D) The expression of the miR-181b in the high pathological level group was lower than that in the low pathological level group in GEO database. (E) Sh-EZH2 increased miR$181 \mathrm{~b}$ expression compared with the NC group. (F) A fragment upstream of human pri-miR-181b stem-loop was constructed and inserted into the luciferase reporter plasmid pGL3. Luciferase assays confirmed that depletion of EZH2 increased the activity of luciferase construction. (G and $\mathrm{H}$ ) Inhibition of miR-181b in EZH2-depleted cells largely abrogated the effect of sh-EZH2 on cellular glucose metabolism. (I) Western blots determined the HK2 expression change following cell transfection with sh-EZH2 and As-miR-181b. ${ }^{*} \mathrm{P}>0.05,{ }^{*} \mathrm{P}<0.05,{ }^{* *} \mathrm{P}<0.01$.

and all identified genes. The EZH2 specific gene signature included 925 gene positively correlated with EZH2 expression, and 1,952 gene negatively correlated with EZH2 expression (Fig. 2A). Among them, a set of glycometabolism-related genes were positively correlated with EZH2 expression, including HK2, solute carrier family 2 member 1 (SLC2A1) and ribosomal protein S6 kinase B1 (RPS6KB1). HK2 was a metabolic enzyme that executes the first step of aerobic glycolysis (Fig. 2B). It was elevated in prostate cancer tissues compared to prostatic hyperplasia tissues. Moreover, its expression significantly increased in CRPC compared to ADPC (Fig. 2C). Therefore, it was chosen for further study. GSEA was used to evaluate the pathways that were differentially expressed between patients with high levels of HK2 expression and those with low levels of HK2 expression. The result revealed that genes correlated with HK2 were primarily associated with glycometabolism and cell cycle progression (Fig. 2D). Glycolysis stress tests demonstrated sh-HK2 significantly inhibited the glycolytic ability of prostate cancer cells (Fig. 3).

miR-181b is an important mediator between EZH2 and HK2. To detect the potential mechanism by which EZH2 regulates
HK2, we performed bioinformatic analysis to identify HK2 as the potential target of miR-181b (Fig. 4A). Western blot analysis showed that HK2 expression was decreased in prostate cancer cells with upregulation of miR-181b (Fig. 4B). To determine the direct interaction between miR-181b and its binding site within HK2 mRNA, we created constructs containing wild-type (pGL3-WT-HK2-3'UTR) and mutant (pGL3-MUTHK2-3'UTR) HK2 3'UTRs. Luciferase reporter assays showed that over-expression of miR-181b led to a marked decrease in luciferase activity of the pGL3-WT-HK2-3'UTR plasmid in PC-3 cells without significant change in luciferase activity of the pGL3-MUT- HK2-3'UTR plasmid (Fig. 4C). Our data provided strong evidence that miR-181b directly regulated HK 2 expression by binding to the 3'UTR of HK2 mRNA in prostate cancer cells.

Next, we found that the expression of the miR-181b in the high pathological level group was lower than that in the low pathological level group in prostate cancer database (Fig. 4D). Then, PC-3 cells were treated with sh-EZH2. miR-181b expression was determined in scrambled and sh-EZH2-treated cells by qRT-PCR. Compared with the scrambled control cells, the sh-EZH2-treated cells showed higher miR-181b expression 
A

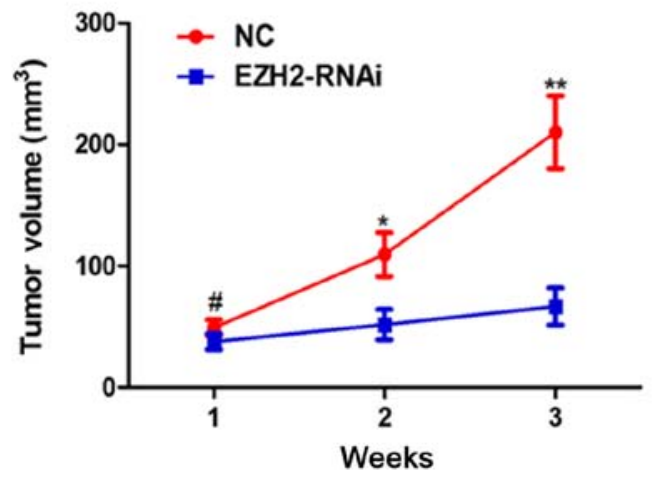

B

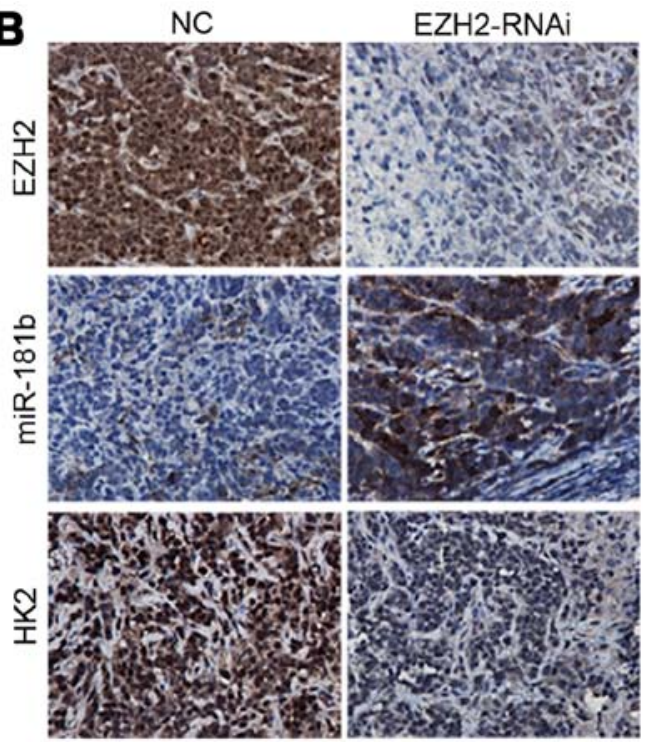

Figure 5. EZH2/miR-181b/HK2 signaling was confirmed in nude mouse tumor xenografts. (A) Nude mice were injected subcutaneously with PC-3 cells infected with EZH2-siRNA-lentivirus or NC. The width and length of tumors were measured every week. ${ }^{~} \mathrm{P}>0.05,{ }^{*} \mathrm{P}<0.05$, ${ }^{* *} \mathrm{P}<0.01$ indicates significant difference when compared to the NC group. (B) IHC and ISH were performed on xenograft tumors. The levels of miR-181b and HK2 were changed in PC-3 xenograft tumors of the EZH2-siRNA-lentivirus group.

(Fig. 4E). A $1.9 \mathrm{~kb}$ fragment upstream of human pri-miR-181b stem-loop was constructed and inserted into the luciferase reporter plasmid pGL3. The plasmid was co-transfected with shEZH2 or NC into PC-3 cells. The depletion of EZH2 increased the activity of luciferase construction (Fig. 4F). These results indicated EZH2 regulated miR-181b expression at transcriptional level in PCa cells. Furthermore, inhibition of miR-181b in EZH2-depleted cells largely abrogated the effect of sh-EZH2 on cellular glucose metabolism and the decreased $\mathrm{HK} 2$ expression in prostate cells, suggesting that EZH2 regulates prostate cancer energy metabolism at least partially in a miR-181b-dependent manner (Fig. 4G-I).

EZH2 inhibition suppresses tumor growth and modulates miR-181b/HK2 axis in vivo. To further investigate the role of $\mathrm{EZH} 2 / \mathrm{miR}-181 \mathrm{~b} / \mathrm{HK} 2$ signaling in tumor growth of prostate cancer in vivo, we extended our investigation by a nude mouse prostate cancer xenograft model. PC-3 cells were infected with EZH2-siRNA-lentivirus. Inhibition of EZH2 repressed prostate cancer xenograft growth in vivo (Fig. 5A). ISH and IHC analysis revealed that miR-181b and HK2 expression were markedly changed following EZH2 inhibition (Fig. 5B).

\section{Discussion}

Prostate cancer is a common malignant tumor in male genitourinary system. Its incidence has obvious geographical and ethnic differences. In the United States, prostate cancer is the most commonly diagnosed cancer and the second leading cause of cancer mortalities in men (19). In Europe, approximately 75,800 patients were estimated to die from prostate cancer in 2016 (20). Its mortality ranks the third in all male malignant tumors. It has become a major health concern in the older male population in the world. At present, radical prostatectomy and external radiation therapy are main methods of treatment for localized prostate cancer. Additionally, hormonal therapy is also an important treatment method for some complicated cases such as patients with distant metastasis or recurrence after treatment. More than $80 \%$ of the patients can alleviate disease through androgen deprivation therapy. However, after 14-30 months, almost all patients with lesions will be gradually transformed to CRPC, which leads to very poor prognosis. Although a variety of drugs are used for CRPC patients, including docetaxel, abiraterone, and enzalutamide, their curative effects are still limited (21). CRPC has become the main cause of death in patients with advanced prostate cancer. It is necessary to further study tumor progression mechanism and novel molecular targets.

$\mathrm{EZH} 2$ is the catalytic member of the polycomb repressive complex 2 (PRC2). It comprises a SET domain which is recognized as the signature of methyltransferases as it provides the active site for the covalent methylation reaction, resulting in trimethylation (me3) of histone 3 (H3) at lysine 27 (K27), as well as at lysine 9 (K9) albeit to a much lesser extent. EZH2 alone exhibits no intrinsic enzymatic function, in order to be catalytically active it must interact with at least two proteins, embryonic ectoderm development (EED) and suppressor of zeste 12 (SUZ12) (22-24).

Studies have found that EZH2 is dysregulated in breast cancer, bladder cancer, gastric cancer, lung cancer, and liver cancer, which indicating poor prognosis $(25,26)$. Also there is higher expression in metastatic prostate cancer tissues than in localized ones $(27,28)$. It was reported that EZH2 promotes the invasive ability of tumor cells through catalyzing $\mathrm{H} 3 \mathrm{~K} 27 \mathrm{me} 3$ and then inhibiting the expression of ADRB2 and CDH1 (29,30). However, the expression of EZH2 is not clear in prostate cancer of different stages and different pathological levels. In addition, we do not know the potential biological function of EZH2 and its relationship with non-coding RNA signaling pathways. In this study, we further evaluated the EZH2 biological behavior in prostate cancer progression and its potential molecular mechanisms by a series of methods 
including clinical sample analysis, bioinformatics, microarray, cell function experiments, molecular biology experiments and animal experiments in vivo. The result indicated that EZH2 expression was elevated in prostate cancer tissues compared to normal prostate tissues.

Further, EZH2 expression significantly increased in CRPC compared to ADPC. Its expression was significantly elevated in Gleason $>7$ prostate cancer tissues compared to Gleason $\leq 7$ ones. EZH2 depletion inhibited cellular colony formation and aerobic glycolysis process in vitro. Additionally, EZH2 inhibition significantly slowed tumor growth rate in nude mouse tumor xenograft experiments. Next, we used gene expression profile microarray to analyze the metabolism-related genes which were correlated with EZH2. The results showed that the expression of HK2 was positively correlated with EZH2. Realtime PCR and luciferase reporter assays showed that EZH2 inversely modulated miR-181b at transcriptional level. Further study indicated that HK2 was a direct target of miR-181b. Moreover, decreased miR-181b expression largely abrogated the effect of sh-EZH2 on HK2 expression and HK2-induced glucose metabolism process. The results indicate that miR$181 \mathrm{~b}$ serves as important mediator between EZH2 and its downstream pathway.

Above all, we discover that EZH2 was able to regulate glucose metabolism in prostate cancer, acquired the EZH2 related gene profile and construct EZH2/miRNA axis and its downstream pathway in prostate cancer. These accomplishments establish a foundation for further research on miRNA-mediated EZH2-related molecule network. Next we are aiming to reveal the network model, providing novel targets and contributing to the optimizing treatment strategies for molecular targeted therapy in prostate cancer.

\section{Acknowledgements}

This study was supported by National Natural Science Foundation of China (81370849, 81300472, and 81572517), and Anhui Natural Science Foundation (1608085MH166 and 1708085QH202).

\section{References}

1. Hanahan D and Weinberg RA: Hallmarks of cancer: The next generation. Cell 144: 646-674, 2011.

2. Vander Heiden MG, Cantley LC and Thompson CB: Understanding the Warburg effect: The metabolic requirements of cell proliferation. Science 324: 1029-1033, 2009.

3. Warburg O: On the origin of cancer cells. Science 123: 309-314, 1956.

4. Kroemer G and Pouyssegur J: Tumor cell metabolism: Cancer's Achilles' heel. Cancer Cell 13: 472-482, 2008.

5. Vander Heiden MG: Targeting cancer metabolism: A therapeutic window opens. Nat Rev Drug Discov 10: 671-684, 2011.

6. Singleterry J, Sreedhar A and Zhao Y: Components of cancer metabolism and therapeutic interventions. Mitochondrion 17: 50-55, 2014.

7. Swietach P, Vaughan-Jones RD and Harris AL: Regulation of tumor $\mathrm{pH}$ and the role of carbonic anhydrase 9. Cancer Metastasis Rev 26: 299-310, 2007.

8. Tao T, Li G, Dong Q, Liu D, Liu C, Han D, Huang Y, Chen S, $\mathrm{Xu} \mathrm{B}$ and Chen M: Loss of SNAIL inhibits cellular growth and metabolism through the miR-128-mediated RPS6KB1/HIF-1 $\alpha /$ PKM2 signaling pathway in prostate cancer cells. Tumour Biol 35: 8543-8550, 2014.

9. Warburg O: On respiratory impairment in cancer cells. Science 124: 269-270, 1956.
10. Yeung SJ, Pan J and Lee MH: Roles of p53, MYC and HIF-1 in regulating glycolysis - the seventh hallmark of cancer. Cell Mol Life Sci 65: 3981-3999, 2008.

11. Hsu PP and Sabatini DM: Cancer cell metabolism: Warburg and beyond. Cell 134: 703-707, 2008

12. Mathupala SP, Ko YH and Pedersen PL: Hexokinase-2 bound to mitochondria: Cancer's stygian link to the 'Warburg Effect' and a pivotal target for effective therapy. Semin Cancer Biol 19: 17-24, 2009.

13. Pedersen PL, Mathupala S, Rempel A, Geschwind JF and Ko YH: Mitochondrial bound type II hexokinase: A key player in the growth and survival of many cancers and an ideal prospect for therapeutic intervention. Biochim Biophys Acta 1555: 14-20, 2002 .

14. Wolf A, Agnihotri S, Micallef J, Mukherjee J, Sabha N, Cairns R, Hawkins C and Guha A: Hexokinase 2 is a key mediator of aerobic glycolysis and promotes tumor growth in human glioblastoma multiforme. J Exp Med 208: 313-326, 2011.

15. Taylor BS, Schultz N, Hieronymus H, Gopalan A, Xiao Y, Carver BS, Arora VK, Kaushik P, Cerami E, Reva B, et al: Integrative genomic profiling of human prostate cancer. Cancer Cell 18: 11-22, 2010.

16. Grasso CS, Wu YM, Robinson DR, Cao X, Dhanasekaran SM, Khan AP, Quist MJ, Jing X, Lonigro RJ, Brenner JC, et al: The mutational landscape of lethal castration-resistant prostate cancer. Nature 487: 239-243, 2012.

17. Zhang C, Zhang J, Hao J, Shi Z, Wang Y, Han L, Yu S, You Y, Jiang $\mathrm{T}$, Wang J, et al: High level of miR-221/222 confers increased cell invasion and poor prognosis in glioma. J Transl Med 10: 119, 2012.

18. Wang XF, Shi ZM, Wang XR, Cao L, Wang YY, Zhang JX, Yin Y, Luo H, Kang CS, Liu N, et al: MiR-181d acts as a tumor suppressor in glioma by targeting K-ras and Bcl-2. J Cancer Res Clin Oncol 138: 573-584, 2012.

19. Siegel RL, Miller KD and Jemal A: Cancer statistics, 2016. CA Cancer J Clin 66: 7-30, 2016.

20. Malvezzi M, Carioli G, Bertuccio P, Rosso T, Boffetta P, Levi F, La Vecchia C and Negri E: European cancer mortality predictions for the year 2016 with focus on leukaemias. Ann Oncol 27: 725-731, 2016.

21. Tao T, Liu D, Liu C, Xu B, Chen S, Yin Y, Ang L, Huang Y, Zhang $\mathrm{X}$ and Chen M: Autoregulatory feedback loop of EZH2/ miR-200c/E2F3 as a driving force for prostate cancer development. Biochim Biophys Acta 1839: 858-865, 2014.

22. Yang YA and $\mathrm{Yu}$ J: EZH2, an epigenetic driver of prostate cancer. Protein Cell 4: 331-341, 2013.

23. Kim J and Yu J: Interrogating genomic and epigenomic data to understand prostate cancer. Biochim Biophys Acta 1825: 186-196, 2012.

24. Varambally S, Cao Q, Mani RS, Shankar S, Wang X, Ateeq B, Laxman B, Cao X, Jing X, Ramnarayanan K, et al: Genomic loss of microRNA-101 leads to overexpression of histone methyltransferase EZH2 in cancer. Science 322: 1695-1699, 2008.

25. Sauvageau M and Sauvageau G: Polycomb group proteins: Multifaceted regulators of somatic stem cells and cancer. Cell Stem Cell 7: 299-313, 2010.

26. Kleer CG, Cao Q, Varambally S, Shen R, Ota I, Tomlins SA, Ghosh D, Sewalt RG, Otte AP, Hayes DF, et al: EZH2 is a marker of aggressive breast cancer and promotes neoplastic transformation of breast epithelial cells. Proc Natl Acad Sci USA 100: 11606-11611, 2003.

27. Berezovska OP, Glinskii AB, Yang Z, Li XM, Hoffman RM and Glinsky GV: Essential role for activation of the Polycomb group $(\mathrm{PcG})$ protein chromatin silencing pathway in metastatic prostate cancer. Cell Cycle 5: 1886-1901, 2006.

28. Hoffmann MJ, Engers R, Florl AR, Otte AP, Muller M and Schulz WA: Expression changes in EZH2, but not in BMI-1, SIRT1, DNMT1 or DNMT3B are associated with DNA methylation changes in prostate cancer. Cancer Biol Ther 6: 1403-1412, 2007

29. Cao Q, Yu J, Dhanasekaran SM, Kim JH, Mani RS, Tomlins SA, Mehra R, Laxman B, Cao X, Yu J, et al: Repression of E-cadherin by the polycomb group protein EZH2 in cancer. Oncogene 27: 7274-7284, 2008

30. Yu J,Cao Q, Mehra R, Laxman B, Yu J, Tomlins SA, Creighton CJ, Dhanasekaran SM, Shen R, Chen G, et al: Integrative genomics analysis reveals silencing of beta-adrenergic signaling by polycomb in prostate cancer. Cancer Cell 12: 419-431, 2007. 